\title{
A FINITE VERSION OF SCHUR'S THEOREM
}

\author{
R. S. KULKARNI 1
}

ABSTRACT. A necessary and sufficient condition for constancy of curvature in terms of umbilicity of small metric spheres is given.

The following is a remark on a paper of Kowalski in which he has investigated the "properties of hypersurfaces which are characteristic for spaces of constant curvature" and papers of Nomizu [3] and Nomizu and Leung [4]. This type of investigation goes back to Schur [5]. For other results of this type we refer to Cartan [1, Chapter V].

We follow the terminology of Kowalski [2]. By a 'metric sphere' in a Riemann manifold $M$ we understand a subset consisting of points at a fixed distance from a fixed point. If the fixed distance is sufficiently small the metric sphere is a smooth hypersurface. Kowalski [2, Theorem 8] shows that if every sufficiently small metric sphere in $M$ is totally umbilic and $\operatorname{dim} M \geq$ 4, then $M$ is conformally euclidean. We shall improve this result as follows:

Theorem. Let $M$ be a connected $C^{\infty}$ Riemann manifold of dimension $\geq 3$. Then every sufficiently small metric sphere is totally umbilic iff $M$ is of constant curvature.

Total umbilicity of metric spheres is, roughly speaking, a finite version of the infinitesimal isotropy condition in Schur's well-known theorem: namely if $M$ is a connected Riemann manifold of dimension $\geq 3$ such that the sectional curvature depends only on the point (and not on the 2-plane section at the point) then the curvature is actually constant. For this reason we have referred to the above theorem as a finite version of Schur's theorem.

Proof of the theorem. First of all note that a space of constant curvature (without any dimension restrictions) has the stated property, cf. e.g., [2, Proposition 5]. (Alternately note that the property is "visibly" true in the Euclidean case, and since umbilicity is a conformally invariant notion, the property also holds in the spherical and hyperbolic cases.)

Let us now prove the converse. Suppose that every small hypersphere of $M$ is totally umbilic. Fix a point $P$ and a unit tangent vector $c_{1}$ at $p$. Let $\gamma$ be geodesic through $p$ tangential to $e_{1}$. Choose a point $q$ on $\gamma, q \neq p$,

Received by the editors October 24, 1974 .

AMS (MOS) subject classifications (1970). Primary 53C20.

${ }^{1}$ Supported in part by National Science Foundation grant GP-36418X. 
sufficiently close to $p$ so that the hypersphere centered at $q$ and passing through $p$ is contained in a normal coordinate neighborhood around $q$. Now choose a moving frame $\left\{e_{1} \cdots e_{m}\right\}$ in a neighborhood of $p$ so that $e_{1}$ is tangential to geodesics through $q$. Let $\left\{\omega_{1}, \cdots, \omega_{m}\right\}$ be the corresponding dual forms. They satisfy the structure equations

(1) $d \omega_{a}=\sum_{b=1}^{m} \omega_{a b} \omega_{b}, d \omega_{a b}=\sum_{c=1}^{m} \omega_{a c} \omega_{c b}+\Omega_{a b}, \quad \omega_{a b}+\omega_{b a}=0$.

We have $\omega_{1}=d r$ where $r$ is the radial parameter measured from $q$. Hence $0=d \omega_{1}=\Sigma_{a=2}^{m} \omega_{1 a} \omega_{a}$. It follows that $\omega_{1 a}$ is a linear combination of $\omega_{2}, \cdots, \omega_{m}$ only. On the other hand, $\omega_{1 a}$ define the second fundamental forms of metric spheres centered at $q$; hence by the total umbilicity of the metric spheres they must be of the form $\omega_{1 a}=\lambda \omega_{a}+\mu_{a} \omega_{1}$, where $\lambda, \mu_{a}$ are smooth functions. Combining these two observations we see that $\mu_{a}=0$ and so

$$
\omega_{1 a}=\lambda \omega_{a}
$$

Taking the exterior derivative and using the structure equations, we have

Consequently

$$
\begin{aligned}
d \omega_{1 a} & =d \lambda \omega_{a}+\lambda \omega_{a 1} \omega_{1}+\sum_{b \geq 2} \lambda \omega_{a b} \omega_{b} \\
& =\sum_{b \geq 2} \omega_{1 b} \omega_{b a}+\Omega_{1 a}=\lambda \sum_{b \geq 2} \omega_{a b} \omega_{b}+\Omega_{1 a} .
\end{aligned}
$$

$$
\Omega_{1 a}=d \lambda \omega_{a}+\lambda \omega_{1} \omega_{1 a}=\left(\partial \lambda / \partial r+\lambda^{2}\right) \omega_{1} \omega_{a}+\text { other terms. }
$$

This shows that at $p$ the sectional curvature of the 2-plane spanned by $e_{1}$ and $e_{a}$ is $-\left(\partial \lambda / \partial r+\lambda^{2}\right)$. Since this expression is independent of $a$, it follows that the sectional curvature of the 2-plane containing $e_{1}$ is the same. Since $e_{1}$ is arbitrary, we see that all sectional curvatures at $p$ are the same. If $\operatorname{dim} M \geq 3$, by the Schur theorem it follows that $M$ is of constant curvature. A further use of Codazzi's equation implies that $\lambda$ is in fact constant on a metric sphere. Q.E.D.

Remark. The above theorem, just as the Schur's theorem, of course, breaks down when $\operatorname{dim} M=2$; in fact any curve on a 2-dimensional manifold is trivially totally umbilic. In this case we can restore the theorem by making a stronger hypothesis.

Theorem. Let $M$ be a 2-dimensional connected Riemann manifold such that every sufficiently small metric sphere is of constant geodesic curvature; then $M$ is of constant curvature.

(In Kowalski's terminology constancy of geodesic curvature of a metric sphere is the same as a metric sphere being a $U$-sphere. So this theorem is 
a partial improvement of [2, Theorem 3]. Converse of course is true and trivial.)

Proof. In the above notation, constancy of geodesic curvature means that the function $\lambda$ is constant on metric spheres-hence it is a function of $r$. So the sectional curvature $-\left(d \lambda / d r+\lambda^{2}\right)$ is constant (say $c$ ) on a metric sphere (say $s$ ). Now considering the metric spheres with centers on $S$, and continuing this way, we see that the set of points where the sectional curvature is $c$ is open and closed. Since $M$ is connected, it must be of constant curvature. Q.E.D.

\section{REFERENCES}

1. E. Cartan, Leçons sur la géométrie des espaces de Riemann, GauthierVillars, Paris, 1963.

2. O. Kowalski, Properties of hypersurfaces, Ann. Sci. Norm. Sup. Pisa 26 (1972), 223-245.

3. K. Nomizu, Generalized central spheres, Tôhoku Math. J. 25 (1973), $129-137$.

4. D. S. Leung and K. Nomizu, The axiom of spheres in Riemannian geometry, J. Differential Geometry 5 (1971), 487-489. MR 44 \#7472.

5. F. Schur, Über den Zusammenhang der Räume konstanten Krümmungsmasses mit den projectiven Räumen, Math. Ann. 27 (1886), 537-567.

SCHOOL OF MATHEMATICS, INSTITUTE FOR ADVANCED STUDY, PRINCETON, NEW JERSEY 08540

Current address: Department of Mathematics, Columbia University, New York, New York 10027 